\title{
HOW LONG DOES IT TAKE TO CONSENSUS IN THE HEGSELMANN-KRAUSE MODEL?
}

\author{
SASCHA KURZ
}

\begin{abstract}
Hegselmann and Krause introduced a discrete-time model of opinion dynamics with agents having limit confidence. It is well known that the dynamics reaches a stable state in a polynomial number of time steps. However, the gap between the known lower and upper bounds for the worst case is still immense. In this paper exact values for the maximum time, needed to reach consensus or to discover that consensus is impossible, are determined using an integer linear programming approach.
\end{abstract}

\section{INTRODUCTION AND PROBLEM FORMULATION}

A Hegselmann-Krause system on the real line, HK system for short, is defined as follows. There are $n$ agents with real-valued initial opinions $x_{1}(0) \leq x_{2}(0) \leq \cdots \leq x_{n}(0)$. For all $t \in \mathbb{N}$ the opinion of agent $i$ at time $t+1$ is given by $x_{i}(t+1)=\left(\sum_{j \in \mathcal{N}_{i}(t)} x_{j}(t)\right) /\left|\mathcal{N}_{i}(t)\right|$, where $\mathcal{N}_{i}(t)=\left\{j:\left|x_{i}(t)-x_{j}(t)\right| \leq 1\right\}$ (cf. [4]). Obviously, the ordering of the agents, i.e., $x_{1}(t) \leq x_{2}(t) \leq \cdots \leq x_{n}(t)$, is preserved over time. We say that a HK system has converged at time $T$ if $x_{i}(t+1)=x_{i}(t)$ holds for all agents and all time steps $t \geq T$. For the convergence time, i.e., the smallest time $T$ such that the system has converged at $T$, an upper bound of $O\left(n^{3}\right)$ was proven [1, 6. It is conjectured that the worst-case scenario is given by the initial positions $x_{i}(0)=i-$ equidistant configuration for later reference - and needs roughly $\frac{4 n}{5}$ time steps [7].
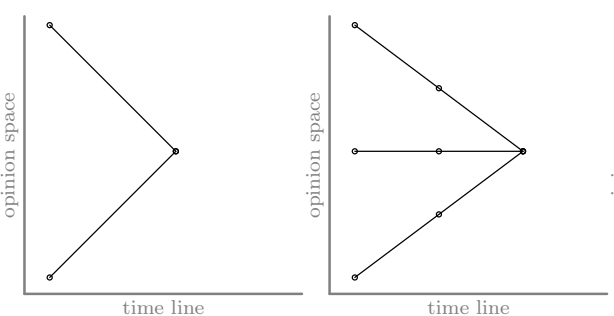
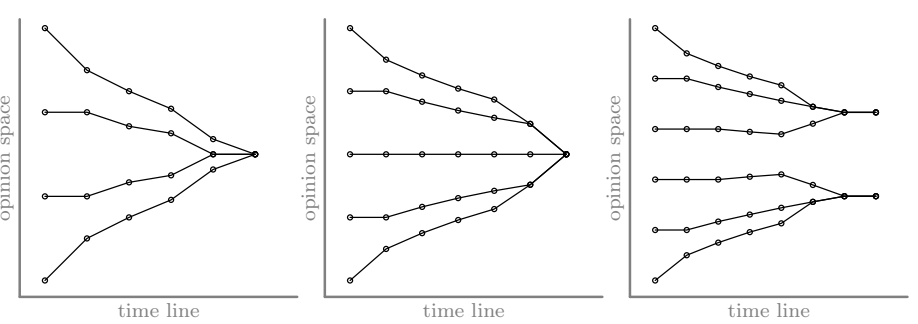

FiguRE 1. Trajectories for the equidistant configuration with $n=2, \ldots, 6$ agents

In an equidistant configuration with at most 5 agents the final state consists of a single opinion, see Figure 1 . There is also the possibility that different clusters arise, as e.g. for $n=6$. We define the weight of $x \in \mathbb{R}$ at time $t$ as $w_{t}(x)=\left\{i: x_{i}(t)=x\right\}$ and the the weight of agent $i$ at time $t$ as $w_{t}\left(x_{i}(t)\right)$. With this, we say that a HK system has reached consensus at time $t$ if the weight of agent 1 equals $n$. Whenever we have $x_{i+1}(t)-x_{i}(t)>1$, we have $x_{i+1}\left(t^{\prime}\right)-x_{i}\left(t^{\prime}\right)>1$ for all $t^{\prime} \geq t$, i.e., no consensus is possible. By $f(\mathcal{C})$ we denote the earliest time $t$ such that a given HK system $\mathcal{C}$ has reached consensus or we have $x_{i+1}(t)-x_{i}(t)>1$ for at least one index $i$. With this let $f(n)$ denote the maximum of $f(\mathcal{C})$ over all configurations consisting of $n$ agents. Clearly $f(\mathcal{C})$ is upper bounded by the convergence time, so that $f(n) \in O\left(n^{3}\right)$.

\section{An ANALYTical LOWER BOUND}

Let $k \geq 4$ be an integer, $j_{1}=1, j_{2}=k+1, j_{3}=k+2, j_{4}=k+3$, and $n=2 k+2$. We consider the HK system $\mathcal{C}$ with initial opinions $x_{i}(0)=-\frac{1}{k}$ for all $1 \leq i \leq k, x_{j_{2}}(0)=0, x_{j_{3}}(0)=1$, and $x_{i}(0)=1+\frac{1}{k}$ for all $j_{4} \leq i \leq n$, i.e., agents $j_{1}$ and $j_{4}$ have weight $k$ and are rather close to agents $j_{2}$ and $j_{3}$, respectively. From the following lemma we conclude $f(\mathcal{C}) \in \Omega(n)$.

Lemma 1. Let $a_{t}=t+1, b_{t}=\frac{(t+1)(t+2)}{2}$, and $c_{t}=t$. For all $0 \leq t \leq \frac{k}{3}$ we have

$$
-\frac{b_{t}}{k^{3}} \leq x_{j_{1}}(t)+\frac{1}{k}-\frac{a_{t}}{k^{2}} \leq 0, \quad 0 \leq x_{j_{2}}(t) \leq \frac{c_{t}}{k^{2}}, \quad 1-\frac{c_{t}}{k^{2}} \leq x_{j_{3}}(t) \leq 1, \quad 0 \leq x_{j_{4}}(t)-1+\frac{1}{k}-\frac{a_{t}}{k^{2}} \leq \frac{b_{t}}{k^{3}} .
$$

Proof. If true, we have $x_{j_{2}}(t)-x_{j_{1}}(t) \leq \frac{c_{t}}{k^{2}}+\frac{1}{k} \leq 1, x_{j_{3}}(t)-x_{j_{2}}(t) \leq 1$, and $x_{j_{3}}(t)-x_{j_{1}}(t) \geq 1-\frac{c_{t}}{k^{2}}+$ $\frac{1}{k}-\frac{a_{t}}{k^{2}}>1$ for all $0 \leq t \leq \frac{k}{3}$, i.e., $\mathcal{N}_{j_{1}}=\left\{1, \ldots, j_{1}, j_{2}\right\}, \mathcal{N}_{j_{2}}=\left\{1, \ldots, j_{1}, j_{2}, j_{3}\right\}, \mathcal{N}_{j_{3}}=\left\{j_{2}, j_{3}, j_{4}, \ldots, n\right\}$, and $\mathcal{N}_{j_{4}}=\left\{j_{3}, j_{4}, \ldots, n\right\}$ due to symmetry. Next we proof the assertion by induction on $t \leq \frac{k}{3}$, where we use $\frac{1}{k}-\frac{1}{k^{2}} \leq \frac{1}{k+1} \leq \frac{1}{k}-\frac{1}{k^{2}}+\frac{1}{k^{3}}$ and $x_{2}(t)+x_{3}(t)=1$ (symmetry again). We can check that for $t=0$ the 
asserted inequalities are satisfied. For $t \geq 0$ we have

$$
\begin{aligned}
x_{j_{1}}(t+1) & =\frac{k \cdot x_{j_{1}}(t)+x_{j_{2}}(t)}{k+1} \leq \frac{k \cdot\left(-\frac{1}{k}+\frac{a_{t}}{k^{2}}\right)+\frac{c_{t}}{k^{2}}}{k+1} \leq\left(\frac{1}{k}-\frac{1}{k^{2}}\right) \cdot\left(-1+\frac{a_{t}}{k}+\frac{c_{t}}{k^{2}}\right) \leq-\frac{1}{k}+\frac{a_{t}+1}{k^{2}}, \\
x_{j_{1}}(t+1) & \geq \frac{k \cdot\left(-\frac{1}{k}+\frac{a_{t}}{k^{2}}-\frac{b_{t}}{k^{3}}\right)+0}{k+1} \geq\left(\frac{1}{k}-\frac{1}{k^{2}}+\frac{1}{k^{3}}\right) \cdot\left(-1+\frac{a_{t}}{k}-\frac{b_{t}}{k^{2}}\right) \geq-\frac{1}{k}+\frac{a_{t}+1}{k^{2}}-\frac{a_{t}+b_{t}+1}{k^{3}}, \\
x_{j_{2}}(t+1) & =\frac{k \cdot x_{j_{1}}(t)+x_{j_{2}}(t)+x_{j_{3}}(t)}{k+2} \geq \frac{k \cdot\left(-\frac{1}{k}+\frac{a_{t}}{k^{2}}-\frac{b_{t}}{k^{3}}\right)+1}{k+2}=\frac{a_{t}-\frac{b_{t}}{k}}{k(k+2)} \geq 0, \text { and } \\
x_{j_{2}}(t+1) & \leq \frac{k \cdot\left(-\frac{1}{k}+\frac{a_{t}}{k^{2}}\right)+1}{k+2}=\frac{a_{t}}{k(k+2)} \leq \frac{a_{t}}{k^{2}}
\end{aligned}
$$

where we always assume $t \leq \frac{k}{3}$ and $k \geq 4$. The remaining inequalities follow from the symmetry of the configuration.

\section{AN INTEGER LINEAR PROGRAMMing MODEL}

By considering $\mathcal{N}_{i}(t) \backslash\{i\}$ as the neighbors of agent $i$, we obtain the influence graph $\mathcal{G}(t)$, which is clearly a unit interval graph. Let $\mathcal{I}_{n}^{c}$ denote the set of connected unit interval graphs with vertex set $V=\{1, \ldots, n\}$, which admit a representation satisfying $x_{i} \leq x_{j}$ for all $i \leq j$, and $K_{n}$ denote the complete graph on $n$ vertices. Given $n, T \in \mathbb{N}$ and $\varepsilon \in \mathbb{R}$ consider the BLP:

$$
\begin{array}{cc}
\min \sum_{I=(V, E) \in \mathcal{I}_{n}^{c}}|E| \cdot u_{I}^{T} & \text { subject to } \\
x_{j}^{t}-x_{i}^{t} \leq 1+\varepsilon+\left(1-u_{I}^{t}\right) \cdot n & \forall 0 \leq t \leq T, I=(V, E) \in \mathcal{I}_{n}^{c}, 1 \leq i<j \leq n \text { with }\{i, j\} \in E \\
x_{j}^{t}-x_{i}^{t} \geq 1-\varepsilon-\left(1-u_{I}^{t}\right) \cdot(1-\varepsilon) & \forall 0 \leq t \leq T, I=(V, E) \in \mathcal{I}_{n}^{c}, 1 \leq i<j \leq n \text { with }\{i, j\} \notin E \\
\sum_{I \in \mathcal{I}_{n}^{c}} u_{I}^{t}=1 \quad \forall 0 \leq t \leq T \quad u_{K_{n}}^{t}=0 \quad \forall 0 \leq t<T & x_{i}^{t}=\sum_{I=(V, E) \in \mathcal{I}_{n}^{c}} \frac{x_{i}^{t-1}+\sum_{\{i, j\} \in E} z_{j, I}^{t-1}}{1+\sum_{\{i, j\} \in E} 1} \quad \forall 1 \leq t \leq T, i \in V \\
z_{i, I}^{t} \leq n \cdot u_{I}^{t} \quad \forall 0 \leq t<T, I \in \mathcal{I}_{n}^{c} i \in V & z_{i, I}^{t} \geq x_{i}^{t}-n \cdot\left(1-u_{I}^{t}\right) \quad \forall 0 \leq t<T, I \in \mathcal{I}_{n}^{c}, i \in V \\
z_{i, I}^{t} \leq x_{i}^{t} \quad \forall 0 \leq t<T, I \in \mathcal{I}_{n}^{c}, i \in V & z_{i, I}^{t} \in[0, n] \quad \forall 0 \leq t<T, I \in \mathcal{I}_{n}^{c}, i \in V \\
u_{I}^{t} \in\{0,1\} \quad \forall 0 \leq t \leq T, I \in \mathcal{I}_{n}^{c} & x_{i}^{t} \in[0, n] \quad \forall 0 \leq t \leq T, i \in V
\end{array}
$$

If it has a solution for $\varepsilon<0$ and $T \geq 1$, then we have $f(n) \geq T+1$. If it has no solution for $\varepsilon \geq 0$, then we have $f(n) \leq T$. By choosing $\varepsilon$ apart from zero we can partially prevent from wrong results, caused by numerical inaccuracies (cf. 3]).

\section{Conclusion AND OPEN PROBlems}

We have defined the maximum time $f(n)$ to a consensus in a HK system of $n$ agents. Our analytical example for the proof of $f(n) \in \Omega(n)$ also shows that it might be hard to decrease the $O\left(n^{3}\right)$ bound for the maximum convergence time, since the known approaches rely on the fact that the leftmost or the rightmost agent moves by at least some $\delta$ in a finite amount of time steps. In our example we have $\delta \in O\left(\frac{1}{n^{2}}\right)$ for $\Omega(n)$ rounds while the distance between two connected agents can be in $\Omega(n)$. Using an integer linear programming model we have determined $f(n)=0,1,2,5,7,9, \geq 12$ for $1 \leq n \leq 7$. It turned out that for at least $n \in\{5,6, \ldots, 14\}$ the equidistant configuration does not lead to the maximum convergence time - disproving a conjecture of [7] 1] Nevertheless we conjecture the maximum convergence time, and so also $f(n)$, to be linearly bounded. Since $\left|\mathcal{I}_{n}^{c}\right|=\left(\begin{array}{c}2 n-2 \\ n-1\end{array}\right) / n \sim \frac{4^{n-1}}{\sqrt{\pi n} \cdot n}$, a column-generation approach might by very beneficial.

The problem becomes even more challenging if we allow the presence of external controls cf. [2, 3, 5].

\section{REFERENCES}

1. A. Bhattacharyya, M. Braverman, B. Chazelle, and H.L. Nguyen, On the convergence of the Hegselmann-Krause system, Proceedings of the 4th conference on Innovations in Theoretical Computer Science, ACM, 2013, pp. 61-66.

2. B. Chazelle, The total s-energy of a multiagent system, SIAM Journal on Control and Optimization 49 (2011), no. 4, 1680-1706.

3. T. Eymann, R. Hegselmann, S. König, S. Kurz, C. Niemann, and J. Rambau, Optimal control in opinion dynamics: Concepts, structures, pitfalls, algorithms, preprint (2014), 30 pages.

4. R. Hegselmann and U. Krause, Opinion dynamics and bounded confidence models, analysis, and simulation, Journal of Artificial Societies and Social Simulation 5 (2002), no. 3, 33 pages.

5. S. Kurz and J. Rambau, On the hegselmann-krause conjecture in opinion dynamics, Journal of Difference Equations and Applications 17 (2011), no. 6, 859-876

6. S. Mohajer and B. Touri, On convergence rate of scalar Hegselmann-Krause dynamics, American Control Conference (ACC), 2013, IEEE, pp. 206-210.

7. E. Wedin and P. Hegarty, The Hegselmann-Krause dynamics for continuous agents and a regular opinion function do not always lead to consensus, arXiv preprint 1402.7184 (2014), 11 pages.

Sascha Kurz, Mathematisches Institut, Universität Bayreuth, 95440 Bayreuth, Germany. E-mail: Sascha.kurz@uni-

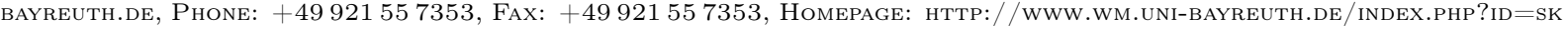

\footnotetext{
${ }^{1}$ Indeed, for $n \geq 2$ the convergence time is given by $1+5\left\lfloor\frac{n+2}{6}\right\rfloor+\frac{1}{3}\left(\sqrt{3} \sin \left(\frac{2 \pi(n-1)}{3}\right)-\cos \left(\frac{\pi(n-1)}{3}\right)-(-1)^{n}\right)$, which tends to $\frac{5 n}{6}$.
} 